Relations industrielles

Industrial Relations

\title{
Assurances sociales
}

\section{La compensation des accidents du travail}

\section{Frédéric-T. Hecker}

Volume 3, numéro 6, février 1948

URI : https://id.erudit.org/iderudit/1023600ar

DOI : https://doi.org/10.7202/1023600ar

Aller au sommaire du numéro

\section{Éditeur(s)}

Département des relations industrielles de l’Université Laval

ISSN

0034-379X (imprimé)

1703-8138 (numérique)

Découvrir la revue

Citer cet article

Hecker, F.-T. (1948). Assurances sociales : la compensation des accidents du travail. Relations industrielles / Industrial Relations, 3(6), 91-95.

https://doi.org/10.7202/1023600ar

Tous droits réservés (C Département des relations industrielles de l’Université Laval, 1948
Ce document est protégé par la loi sur le droit d'auteur. L'utilisation des services d'Érudit (y compris la reproduction) est assujettie à sa politique d'utilisation que vous pouvez consulter en ligne.

https://apropos.erudit.org/fr/usagers/politique-dutilisation/ 
a buyer and a seller. Employer and workers will be in some way associated. The Structure thus, will correspond to an ideal of reconciliation, of fusion, and of social unity. There is no doubt that the results will be good. It is not the place of the Province of Quebec to give on the North American continent, public witness of the solid worth of the Social Doctrine of the Church ?
People say:-« But - who ? 》

The Pope answers: «Undoubtedly the first and immediate apostles of the workingmen must themselves be workingmen, while the apostles of the industrial and commercial world should themselves be employers and merchants. ${ }^{10}$

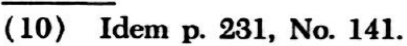

ASSURANCES SOCIALES

\title{
LA COMPENSATION DES ACCIDENTS DU TRAVAIL
}

\author{
Frédéric-T. HECKER
}

Le but de la présente Loi des accidents du travail qui est en vigueur depuis 1931, est de pourvoir à une réparation plus efficace en faveur des ouvriers blessés à leur travail, d'éviter les litiges et d'assurer le service prompt et exact des presta. tions à l'ouvrier et ses dépendants sans obérer indûment l'employeur.

La Loi offre égale protection à l'ouvrier pour tous les accidents survenus par le fait ou à l'occasion de son travail au lieu de s'appliquer seulement à ceux qui sont dus à la négligence du patron; elle attribue le règlement des réclamations à la Commission des Accidents du Travail aux lieu et place des tribunaux; et dans la plupart des industries auxquelles la Loi s'applique, elle rend la responsabilité patronale collective au lieu d'individuelle.

\section{Application de la Loi}

La Loi ne s'applique pas à toutes les industries, mais seulement à celles énumérées dans les Cédules 1 et 2.

La Commission perçoit des cotisations des employeurs dirigeant les entreprises énumérées dans la Cédule 1; et ces cotisations, sous un système de responsabilité collective, constituent le fonds d'accident à même lequel sont payées les compensations et l'assistance médicale. Dans le cadre beaucoup plus restreint des industries énumérées dans la Cédule 2, chaque employeur est tenu personnellement de payer la compensation et de pourvoir à l'assistance médicale en faveur de ses ouvriers blessés par suite des accidents.

Des actions peuvent encore être intentées devant les tribunaux à l'encontre d'employeurs dont les industries ne sont pas assujetties à la Loi en établissant la négligence ou l'incurie de ces derniers, et la présente Loi n'enlève aucun des recours de droit commun appartenant aux personnes qui ne sont pas assujetties à ses dispositions.

\section{Quand la compensation est payable}

La compensation est payable en réparation de toute blessure corporelle résultant d'un accident survenu par le fait ou à l'occasion du travail, et dans les cas de certaines maladies énumérées dans la Cédule 3 de la Loi.

Les seules exceptions à la règle générale sont:

(1) Lorsque l'incapacité résultant de l'accident dure moins de sept jours;

(2) Lorsque l'accident est uniquement imputable à l'imprudence grossière et volontaire de l'ouvrier et qu'il n'entraîne ni la mort ni l'incapacité grave de la victime.

Le fait de la négligence ou de labsence de négligence de l'employeur ou de l'ouvrier ne change pas la situation, et les moyens de défense basés sur la loi civile ordinaire ne s'appliquent pas. 
Aucune entente pour se soustraire à la Loi n'est valide; aucune contribution pour soulager l'employeur de ses responsabilités sous la Loi ne peut être perçue ou collectée de l'ouvrier; la compensation est incessible et insaisissable.

Les compensations payables sous la Loi remplacent le droit d'action en dommages prévu dans la loi civile et aucune action n'est reçue devant une cour de justice en recouvrement d'aucun des bénéfices ou avantages déterminés dans la Loi des Accidents du Travail.

\section{La compensation dans les cas de décès}

Lorsqu'un accident cause la mort d'un ouvrier, la veuve a droit à une rente de $\$ 45.00$ par mois, à compter du ler juillet 1947, et ce, tant qu'elle restera en veuvage. Si l'ouvrier laisse aussi des enfants, la veuve recevra de plus $\$ 10.00$ par mois pour chaque enfant âgé de moins de 18 ans, - la rente des enfants devant être portée à $\$ 15.00$ dans le cas où la veuve décéderait - mais le montant total de la rente mensuelle payable ne doit pas excéder les deux-tiers des gains mensuels moyens de l'ouvrier. Toutefois, l'insuffisance des salaires de l'ouvrier ne réduira pas la rente de sa veuve et d'un enfant à un montant inférieur à $\$ 55.00$ par mois. Une veuve ayant plus d'un enfant recevra une rente minimum de $\$ 65.00$ par mois. La veuve a aussi droit à une allocation spéciale de $\$ 100.00$.

Lorsque l'ouvrier ne laisse pas de veuve, mais seulement des enfants, chacun de ceux-ci a droit à $\$ 15.00$ par mois jusqu'à ce qu'il ait atteint l'âge de 18 ans, mais le montant total de la rente mensuelle ainsi payée ne peut en aucun cas excéder les deux tiers des gains mensuels moyens de l'ouvrier.

Lorsque l'ouvrier ne laisse ni veuve ni enfants, mais d'autres dépendants, ceux-ci ont droit à une allocation raisonnable et proportionnée à la perte pécuniaire subie par chacun de ces dépendants à la suite de la mort de l'ouvrier, mais dans aucun cas cette compensation ne doit excéder les deux tiers des gains mensuels moyens de l'ouvrier.

Il y a des disposition spéciales concernant les enfants âgés de plus de 18 ans qui sont invalides.

Si la veuve contracte un mariage subséquent, sa rente se termine avec ce mariage, mais elle a droit à un montant final équivalent à sa rente mensuelle pendant une période de deux ans. Les paiements en faveur des enfants seront continués.

Les frais funéraires résultant du décès sont payables sous la Loi jusqu'à concurrence d'un montant n'excédant pas $\$ 175.00$.
Pour illustrer ces dispositions de la Loi, prenons le cas d'un ouvrier qui laisse une veuve et trois enfants âgés de moins de 18 ans. La veuve recevra une allocation spéciale de $\$ 100.00$ et une pension de $\$ 45.00$ par mois pour elle-même tant que durera son veuvage et, en plus, $\$ 10.00$ par mois pour chaque enfant tant qu'il sera âgé de moins de 18 ans, et les frais funéraires tel que mentionné ci-haut. Toutefois, si les pensions prévues excèdent les deux tiers des gains mensuels moyens de l'ouvrier, elles seront sujettes à réduction suivant les explications déjà fournies.

\section{La compensation dans les cas d'incapacité}

Un ouvrier totalement incapable de travailler doit recevoir les deux-tiers (66 2-3\%) de la moyenne de ses gains hebdomadaires (sans tenir compte de tout ce qui excède un salaire annuel de $\$ 2$,500.00); mais, en aucun cas ne doit-il recevoir moins que $\$ 15.00$ par semaine, à moins que la moyenne de ses gains hebdomadaires soit inférieure à ce montant, et alors il recevra un montant égal à ses gains moyens.

Ainsi, un ouvrier dont les salaires hebdomadaires moyens ont été de $\$ 24.00$, à la suite d'un accident, recevra une compensation de $\$ 16.00$ par semaine; un ouvrier gagnant $\$ 33.00$ recevra $\$ 22.00$; un ouvrier gagnant $\$ 20.00$ recevra $\$ 15.00$, et un ouvrier gagnant des salaires hebdomadaires moyens de $\$ 10.00$ recevra $\$ 10.00$ par semaine.

La compensation est payable à compter du jour de l'incapacité, mais aucune compensation n'est payable lorsque l'incapacité dure moins de sept jours de calendrier.

Si l'ouvrier n'est affligé que d'une incapacité partielle, la compensation sera équivalente à la même fraction de l'indemnité due pour incapacité totale que celle représentant la différence entre son état après l'accident et sa pleine capacité de gains avant d'être blessé.

La compensation durant la période d'incapacité temporaire est payée à tous les quinze jours.

Après que l'ouvrier aura reçu ses compensations, à tous les quinze jours, durant la période de la consolidation de la blessure, il y aura lieu de considérer l'incapacité permanente résultant de l'accident. Les compensations payables pour l'incapacité permanente, totale ou partielle, seront versées sous forme d'une rente viagère, sauf si l'incapacité permanente n'excède pas $10 \%$. Dans ce dernier cas, la compensation est payée en un versement unique ou par versements périodiques limités. 
Ainsi, un ouvrier dont les salaires moyens ont été de $\$ 120.00$ par mois, affligé d'une incapacité permanente le privant de la moitié de ses pouvoirs de gains, recevra une rente à vie de $\$ 40.00$ par mois, représentant les deux-tiers de la moitié de $\$ 120.00$. Si son incapacité est totale et permanente, il recevra, sa vie durant, une rente de $\$ 80.00$ par mois.

\section{La fixation des compensations}

Toute question se rapportant au droit à la compensation et au montant de celle-ci est du ressort exclusif de la Commission et de ses officiers, la procédure et les méthodes employées étant les plus simples, les plus expéditives et les moins coùteuses.

L'ouvrier doit aviser sans délai son employeur de l'accident qu'il a subi, et l'employeur, dans tous les cas impliquant le paiement d'une compensation, ou de l'assistance médicale, doit en informer la Commission dans un délai de huit jours.

Des affiches, indiquant les droits de l'ouvrier, et rappelant les devoirs de l'ouvrier et de l'employeur lorsqu'un accident survient sont fournies par la Commission et doivent être placées par l'employeur dans un endroit où elles seront vues de tous.

Des formules contenant les détails sur un accident doivent être remplies par l'ouvrier blessé, par l'employeur et par le médecin. Ces formules seront fournies, sur demande, aux employeurs et des formules destinées à l'usage de l'ouvrier et du médecin sont expédiées par la Commission aux parties intéressées dès qu'elle ést informée qu'un accident s'est produit. Si un ouvrier blessé ne reçoit pas ses formules immédiatement, il devrait écrire à la Commission.

La Commission négocie directement avec les intéressés : il n'est pas nécessaire d'avoir recours à aucune assistance légale.

Si les renseignements fournis sur les rapports reçus de l'ouvrier, de l'employeur et du médecin sont suffisants, le premier chèque sera émis généralement dans les deux jours qui suivent la réception du dernier de ceux-ci. Si ces renseignements sont insuffisants, des détails additionnels seront demandés, ou, suivant le cas, une enquète sera entreprise par un officier de la Commission, et au besoin des dépositions seront reçues sous serment devant cet officier ou par la Commission elle-même.

Des rapports additionnels sur l'état de santé de l'accidenté seront demandés à différents intervalles, et, si les rapports fournis le justifient, les paiements bi-mensuels seront faits dès qu'ils seront dus.

Dans les cas d'incapacité permanente, des rapports spéciaux seront demandés de l'ouvrier blessé, de l'employeur et du médecin.

Dans tous les cas où il y a nécessité, l'ouvrier blessé sera soumis à un examen médical devant un expert ou un médecin de la Commission.

Un avis de chaque paiement ou adjudication ( sauf des paiements de pensions à la suite d'une décision) est adressé à l'employeur le jour suivant l'émission.

Lorsqu'un ouvrier a reçu de son employeur un paiement ou une avance sur la compensation qui lui est due, durant la période d'incapacité, le montant ainsi payé ou avancé est déduit des indemnités et peut être remboursé à l'employeur.

\section{II}

\section{L'assistance médicale}

Lorsqu'une réclamation est acceptée par la Commission, quelle que soit la durée de l'incapacité, l'ouvrier a droit à l'assistance médicale, chirurgicale et dentaire; il a droit aussi à l'hospitalisation et aux services de gardes-malades diplômées, et, à la discrétion de la Commission, si l'ouvrier est rendu incapable de façon totale et permanente, à tous les autres traitements, services et assistance que son état peut requérir; l’on doit lui fournir les membres artificiels et autres appareils, les prothèses et autres appareils dentaires qui sont nécessaires par suite de l'accident. Ces membres, instruments ou appareils doivent être gardés en bon état d'usage et seront réparés ou remplacés chaque fois que, dans l'opinion de la Commission, la chose est nécessaire. Tous ces soins et fournitures sont compris sous la rubrique «assistance médicale ».

Dans les cas des industries mentionnées à la Cédule 1 de la Loi, l'assistance médicale est payée par et sous la direction de la Commission, tandis que pour les industries de la Cédule 2, elle sera fournie et payée par l'employeur; dans tous les cas, toute question ou dispute s'y rapportant sera décidée par la Commission.

Sous la Loi, l'ouvrier blessé a le libre choix de son médecin traitant, mais il devrait obtenir de l'employeur un billet ou mémoire indiquant son nom et la date de laccident pour remettre à son médecin.

L'ouvrier doit, à la demande de son employeur, se soumettre à l'examen d'un médecin désigné par cet employeur, et il doit en outre, s'il en 
est requis par la Commission, se soumettre à l'examen du médecin choisi par celle-ci.

Un ouvrier ne peut, sans la permission ou l'approbation de la Commission, laisser la province ou se rendre à distance pour recevoir des traitements ou changer le médecin qu'il a librement choisi.

Lorsqu'une réclamation a été acceptée par la Commission, la Loi ne permet pas à l'employeur de percevoir ou de retenir de l'ouvrier aucune contr1bution pour payer l'assistance médicale fournie; le médecin ne peut faire aucune collection auprès de l'ouvrier pour des soins qu'il a donnés sous l'empire de la Loi. Les honoraires payés par la Commission sont établis suivant un tarif approuvé par la profession médicale.

\section{Premiers secours et ambulance}

Suívant le nombre d'ouvriers à leur emploi, les employeurs sont tenus, par des règlements adoptés sous la Loi, de placer dans leurs usines ou entreprises tout l'équipement nécessaire aux premiers secours. Ils doivent aussi fournir à leurs frais, aux ouvriers blessés, et suivant que leur état le requiert, les services d'une ambulance ou autre moyen de transport chez le médecin, à l'hôpital, ou au domicile de la victime.

\section{Réhabilitation}

La Loi pourvoit à ce que la Commission prenne les mesures et encoure les dépenses nécessaires ou appropriées à la réhabilitation des ouvriers blessés. A cette fin, depuis le mois de décenıbre 1944, la Commission a organisé au sein de ses services administratifs, un Département chargé spécialement de la réhabilitation. Au cours de l'année 1947, une clinique médicale a été fondée où les victimes d'accidents reçoivent des traitements destinés à promouvoir leur rétablissement et à hâter leur retour à l'emploi. Un grand nombre d'ouvriers affligés d'incapacité permanente seront ainsi rétablis dans des occupations où ils pourront gagner leur vie.

\section{Le fonds d'accident}

Le fonds d'accident, à même lequel sont payées les compensations et l'assistance médicale pour les industries de la Cédule 1, est formé des cotisations perçues, suivant un mode ressemblant à la taxe municipale, et qui sont basées au pourcentage de la liste de paye de l'employeur. Les montants perçus doivent être suffisants pour rencontrer les frais des accidents survenus dans la classe à laquelle appartient l'industrie concernée. Tout employeur dirigeant une industrie mentionnée dans la Cédule 1 de la Loi doit, sans avis préalable, préparer et transmettre à la Commission, avant le 20 janvier de chaque année, un état des salaires qu'il a effectivement payés dans l'année précédente, et un estimé des salaires qu'il croit devoir payer durant l'année en cours; tout employeur qui débute dans l'industrie au cours d'une année doit la rapporter à la Commission sans délai. Un état précis des salaires payés doit être tenu.

La cotisation annuelle est d'abord établie provisoirement au début de l'année suivant l'estimé des salaires qui seront payés, et subséquemment, à la fin de l'année, la cotisation sera rajustée selon les salaires effectivement payés durant cette même année. Le taux de la cotisation sera aussi modifié suivant que l'expérience accidentelle de la classe ou du groupe d'industries le justifiera.

Les employeur dans la Cédule 2 seront cotisés, en paiement de la proportion des accidents survenus dans leurs industries, pour les frais d'administration seulement, mais ne contribuent point autrement au fonds d'accident.

\section{Avantages du système actuel}

La présente Loi et les méthodes d'administration offrent de grands avantages sur l'ancienne législation et sur d'autres systèmes d'application.

Sous l'ancienne loi, un ouvrier blessé devait intenter une action en dommages et devait prouver que son employeur avait été coupable de négligence, et que lui-même, la victime de l'accident, n'était pas en faute.

Sous la loi actuelle, la victime et ses dépendants ne reçoivent pas de réparation pour dommages causés, mais bien une compensation, et l'on évalue que vingt fois plus d'ouvriers blessés et leurs dépendants reçoivent des indemnités que l'on n'accordait des dommages aux demandeurs sous l'ancien système. Le principe que l'on applique maintenant c'est de pourvoir aux besoins d'un ouvrier blessé plutôt que de pénaliser l'employeur.

Quand un ouvrier est blessé à son travail et si l'accident cause sa mort, c'est une question de justice aussi bien que d'équité qu'une compensation soit offerte en réparation. C'est aussi une question de droit et non matière à charité, mais la Loi stipule que chaque réclamation doit être considérée d'après le mérite réel et la justice du cas; la Commission n'est pas tenue de suivre les règles ordinaires de la preuve en matière civile. Des instances judiciaires et l'intervention des avocats, avec 


\section{3è CONGRÈS DES RELATIONS INDUSTRIELLES DE LAVAL \\ CHÂtEAU FRONTENAC, QUÉBEC \\ les lundi et mardi, 19 et 20 avril 1948}

\section{Pragramene}

\section{LUNDI, 19 AVRIL 1948}

\section{Avant-midi}

Président: M. C.-N. Moisan, président et gérant-général de Standard Paper Box Ltd et membre du Conseil exécutif national de l'Association des manufacturiers canadiens, inc., membre du Conseil supérieur du Travail.

9.00 Inscription.

9.30 Allocutions de Mgr Ferdinand Vandry, recteur de l'Université Laval et de M. Gérard Tremblay, directeur du Département des relations industrielles.

10.00 Formes de collaboration patronale ouvrière: convention collective. - Forum.

M. Philippe Lessard, président de la Fédération nationale des travailleurs de la pulpe et du papier, inc.

Après-midi

Président: M. J.-A. Juneau, président de l'Association des marchands détaillants du Canada, inc.

2.30 Formes de collaboration patronale-ouvrière: formation professionnelle. - Forum.

M. Gabriel Rousseau, conseiller technique du Service de l'apprentissage du ministère du Travail.
MARDI, 20 AVRIL 1948

\section{Avant-midi}

Président: M. Joseph Matte, échevin de la cité de Québec et membre du Conseil supérieur du Travail.

9.30 Formes de collaboration patronale-ouvrière: comités d'entreprises. - Forum.

M. Raymond Gérin, M.Sc.S., secrétaire de la régionale de Québec de l'Association professionnelle des industriels.

\section{Après-midi}

Président: Docteur Bertrand Bellemarre, médecin hygiéniste industriel à la Commission des accidents du travail.

2.30 Formes de collaboration patronale-ouvrière: sécurité du travail, médecine et hygiène industrielles. Forum.

Docteur Wilfrid Leblond, professeur à la Faculté des sciences sociales.

6.30 Dîner de clôture sous la présidence d'honneur de l'honorable Antonio Barrette, ministre provincial du Travail.

Conférencier invité: T.R.P. Georges-H. Lévesque, o.p.

Les frais d'inscription sont de $\$ 10.00$ et comprennent la participation aux conférences et au dîner de clôture de même qu'ils donnent droit de recevoir le rapport des assises. En raison du nombre limité des congressistes, il est recommandable de faire parvenir son adhésion au plus tôt au secrétariat du Département des relations industrielles, 2 rue de l'Université, Québec.

appel d'un tribunal à l'autre, représenteraient beaucoup moins d'argent pour l'ouvrier et plus de frais pour l'employeur.

\section{Résultats obtenus \\ C.. R}

La Loi fournit à des milliers d'ouvriers, à leurs veuves et à leurs enfants, dans toute les parties de la Province, des secours et de l'assistance sans lesquels les bénéficiaires seraient dans l'indigence. Depuis la mise en vigueur de la Loi, jusquà la fin de 1946, le nombre des accidents dont la Commission a disposé s'élève à 975,083 , et une somme de $\$ 84,457,882.00$ a été payée en compensation.

La Commission reçoit en moyenne 300 rapports d'accidents chaque jour. Environ 800 chèques sont expédiés quotidiennement aux accidentés et plus de 300 comptes sont payés tous les jours pour les frais d'assistance médicale. Pour payer ces compensations et cette assistance médicale, des cotisations sont perçues de plus de 18,000 employeurs.

\section{Conclusions}

Après seize années d'expérience sous l'empire de la présente Loi, l'on croit que peu d'ouvriers ou d'employeurs voudraient revenir aux anciennes méthodes. Les faits saillants de la mise en application de cette législation sont la simplicité de ses dispositions et de la procédure qu'elle prévoit, la disparition des litiges et des dépenses qu'ils entraînaient, une plus grande protection pour l'ouvrier et ses dépendants, l'immunité de l'employeur contre la responsabilité individuelle, et le paie. ment expéditif des compensations versées directement à l'ouvrier ou à ses dépendants. 